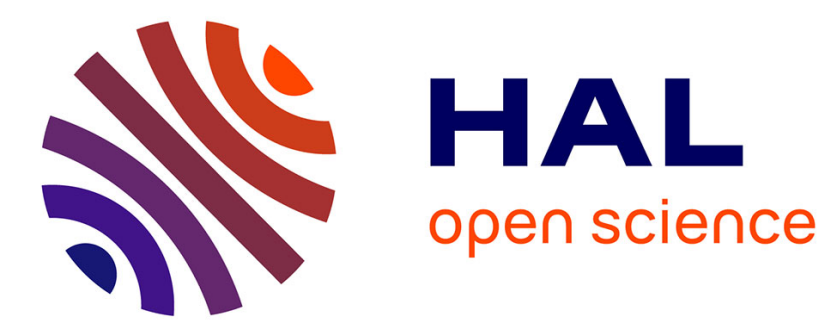

\title{
Coherent quantum dynamics launched by incoherent relaxation in a quantum circuit simulator of a light-harvesting complex
}

\author{
Alex Chin, Etienne Mangaud, O. Atabek, M. Desouter-Lecomte
}

\section{To cite this version:}

Alex Chin, Etienne Mangaud, O. Atabek, M. Desouter-Lecomte. Coherent quantum dynamics launched by incoherent relaxation in a quantum circuit simulator of a light-harvesting complex. Physical Review A, 2018, 97 (6), 10.1103/PhysRevA.97.063823 . hal-02372604

\section{HAL Id: hal-02372604 https://hal.sorbonne-universite.fr/hal-02372604}

Submitted on 20 Nov 2019

HAL is a multi-disciplinary open access archive for the deposit and dissemination of scientific research documents, whether they are published or not. The documents may come from teaching and research institutions in France or abroad, or from public or private research centers.
L'archive ouverte pluridisciplinaire HAL, est destinée au dépôt et à la diffusion de documents scientifiques de niveau recherche, publiés ou non, émanant des établissements d'enseignement et de recherche français ou étrangers, des laboratoires publics ou privés. 


\title{
Coherent quantum dynamics launched by incoherent relaxation in a quantum circuit simulator of a light-harvesting complex
}

\author{
A. W. Chin, ${ }^{1,2}$ E. Mangaud, ${ }^{2,3}$ O. Atabek ${ }^{4}$ and M. Desouter-Lecomte ${ }^{1,5}$ \\ ${ }^{1}$ Laboratoire Chimie Physique (LCP)-CNRS, Université Paris Saclay, Université Paris Sud, F-91405 Orsay, France \\ ${ }^{2}$ Institut des NanoSciences de Paris, Sorbonne Université, 4 place Jussieu, boite courrier 840, 75252 Paris Cedex 05, France \\ ${ }^{3}$ Laboratoire Collisions Agrégats Réactivié (IRSAMC), Universié Toulouse III Paul Sabatier, UMR 5589, F-31062 Toulouse Cedex 09, France \\ ${ }^{4}$ Institut des Sciences Moléculaires d'Orsay (ISMO) UMR CNRS 8214, Université Paris Saclay, Université Paris Sud, F-91405 Orsay, France \\ ${ }^{5}$ Département de Chimie, Université de Liège, Sart Tilman B6, B-4000 Liège, Belgium
}

(Received 30 March 2018; published 12 June 2018)

\begin{abstract}
Engineering and harnessing coherent excitonic transport in organic nanostructures has recently been suggested as a promising way towards improving manmade light-harvesting materials. However, realizing and testing the dissipative system-environment models underlying these proposals is presently very challenging in supramolecular materials. A promising alternative is to use simpler and highly tunable "quantum simulators" built from programmable qubits, as recently achieved in a superconducting circuit by Potočnik et al. [A. Potočnik et al., Nat. Commun. 9, 904 (2018)]. We simulate the real-time dynamics of an exciton coupled to a quantum bath as it moves through a network based on the quantum circuit of Potočnik et al. Using the numerically exact hierarchical equations of motion to capture the open quantum system dynamics, we find that an ultrafast but completely incoherent relaxation from a high-lying "bright" exciton into a doublet of closely spaced "dark" excitons can spontaneously generate electronic coherences and oscillatory real-space motion across the network (quantum beats). Importantly, we show that this behavior also survives when the environmental noise is classically stochastic (effectively high temperature), as in present experiments. These predictions highlight the possibilities of designing matched electronic and spectral noise structures for robust coherence generation that do not require coherent excitation or cold environments.
\end{abstract}

DOI: 10.1103/PhysRevA.97.063823

\section{INTRODUCTION}

Creating and sustaining "long-lived" electronic coherences in complex, multicomponent supramolecular systems has recently been highlighted as an exciting route towards advanced molecular nanodevices, with applications ranging from energy harvesting to optomechanics and sensing [1,2]. In this context, long lived refers to decoherence times of comparable duration to the "functional" timescales of the system, which might, for example, correspond to energy-transport times or charge generation, in the case of photovoltaic light-harvesting structures [3-11]. However, many-if not all-reliable molecular functions are driven in a thermodynamic direction by noisy interactions between electronic degrees of freedom and their thermal environments, so complete suppression of environmental couplings - as is desirable for quantum computationis not a fruitful strategy for the multitude of applications discussed in Refs. [1,2].

Instead, there has been an emerging interdisciplinary focus on understanding how it may be possible to exploit the nonperturbative and non-Markovian dynamics of structured system-environment interactions in nanostructured systems [3,4,9,12-22], with mounting theoretical evidence that a transient and correlated interplay of dissipative and coherent dynamics may be advantageous for a wide range of ultrafast optoelectronic processes. Indeed, although this essential idea has an origin in studies of photosynthetic pigment-protein complexes (PPCs), it is in rationally designed, organic functional materials, such as DNA origami, polymer-fullerene heterojunctions, carbon nanotubes, and molecular dimer systems, that the existence and potentially beneficial impacts of electronic coherence and "noise-assisted" dynamics on light-harvesting processes have been most cleanly and recently demonstrated [23-29]. Examples of theoretically proposed noise-assisted quantum phenomena and their potential applications are reviewed in Refs. [1,2,8].

Regardless of whether Nature got there first, or at all [30], these latter studies underscore the new possibilities arising from exploiting emerging nanofabrication techniques to tune both the properties of photoexcited states (delocalization, dipoles, energy spectrum) and their environments to obtain novel optoelectronic materials based on tailored system-environment interactions. Recently, Potočnik et al. have demonstrated the first experimental "quantum simulator" of an open quantum light-harvesting model built from transmon qubits in a superconducting circuit (Fig. 1) [31]. Using three individually tunable qubits coupled to a transmission line (for photoexcitation) and a resonator (to detect emission), Potočnik et al. demonstrated the formation of robust, delocalized photoexcited states with optical properties analogous to the Davydov-split (Frenkel) excitonic states found in PPCs or J-aggregates $[32,33]$. These states are engineered, as in photosynthetic antennae complexes, so that energy absorbed by the highest-energy state is spatially directed by dissipation towards the lowest-energy state [33,34], which is proximate to a "reaction center" that transduces this incoming energy (here, the resonator). Although impossible in any real 
(a)

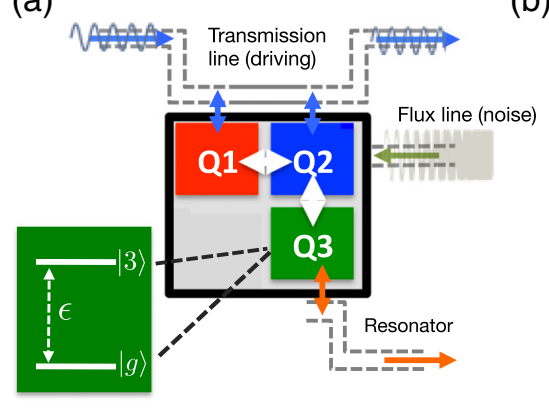

(b)

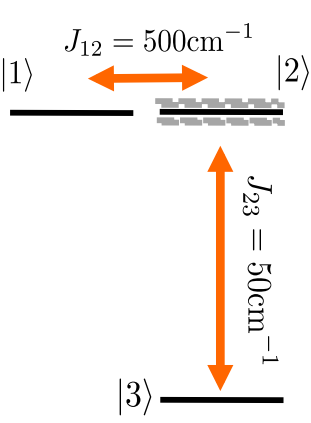

(c)

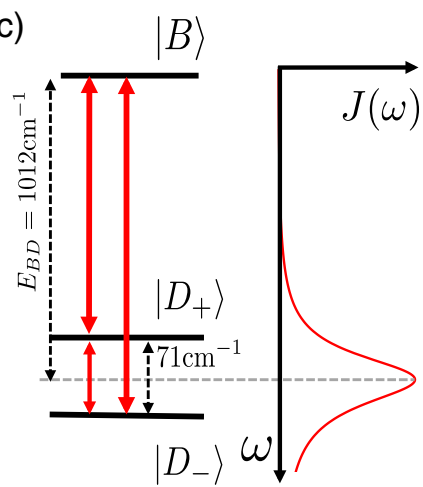

FIG. 1. (a) A schematic representation of the superconducting quantum circuit used in Ref. [31] to simulate energy transport in a photosynthetic light-harvesting array. Here, three qubits $(Q 1-Q 3)$ act as chromophores with a tunable excitation energy $\epsilon_{i}$ and are coupled together by nearest-neighbor capacitive interactions (white arrows). Qubits 1 and 2 are coupled identically to a transmission line which carries the excitation and pump fields, while only emission in the resonator line is uniquely sensitive to the excitation of qubit 3 . The flux lines are used to tune $\epsilon_{i}$, allowing the application of stochastic signals to mimic an arbitrary classical dephasing noise on the qubit (chromophore) system. (b) The rescaled electronic couplings and detunings of the individual qubits and sites used in [31] and in this paper. (c) The resulting spectrum of the bright $|B\rangle$ and dark $\left|D_{ \pm}\right\rangle$eigenstates and the structured (peaked) spectral noise density investigated in the experiment and in this paper.

supramolecular structure, this setup also allows controllable application of environmental dephasing noise of arbitrary strength and spectral properties, which in the basis of delocalized states (vide infra) leads to controllable incoherent transitions between the single-particle excited states of the network. This feature of the experiment makes it a near-ideal platform for testing theories of open dynamics, and by varying the noise coupling strength, it was demonstrated that the energy transferred across the network was maximized at an optimal value of the dephasing rate, precisely as predicted by recent theories of "noise-assisted transport" (also known as ENAQT) [35-38]. In further agreement, this optimal dephasing noise strength was found to be of similar magnitude to the smallest coherent coupling between the qubits, occurring at the "strongto-weak-coupling" transition point where the lowest-energy delocalized eigenstates begin to collapse into effectively localized on-site excitations with sequential hopping transport [31].

Additionally, they also confirmed that energy transfer is considerably more efficient when the spectral function of the environment is strongly peaked around the energy differences between the excitonic excited states. Such structured environmental spectral functions are characteristic of molecular vibrations, and have become intensively studied in open quantum system theory due to their multiscale linear response functions (memory effects) and often nonperturbative coupling to the excited states at specific frequencies. These properties allow potentially qualitative and nonstationary modifications of excited-state dynamics (cf. simple heat baths) that have been connected to (transient) phenomena such as violation of detailed balance, extension of electronic coherence times, and vibronic mixing of electronic states $[3,4,7,16,18,19,21,22,26,39-41]$. However, to describe the dissipative dynamics of systems coupled to such environments requires advanced numerical and analytical techniques, and approaches ranging from many-body methods to advanced master-equation formulations have recently been applied or developed for this aim [12,17,42-55].

In this article, we explore the real-time dynamics of the three-qubit model implemented by Potočnik et al, using the numerically exact hierarchical equations of motion (HEOM) technique [54,56-61] to address a number of theoretical questions that could be verified in a future time-resolved version of the experiment. Specifically, we shall show that the setup of Ref. [31] is an ideal platform to demonstrate the generation of coherence by incoherent processes, in this case caused by the noise-induced relaxation of a high-energy state into a closely spaced doublet of lower-energy states. This is a timely topic, as most observations of coherent optoelectronic phenomena only appear under coherent excitation, whereas functional light-harvesting devices are likely to operate under incoherent illumination, i.e., sunlight $[62,63]$. Without access to excitation sources that can generate electronic coherences, i.e., laser pulses, future coherent devices must rely on transient internal or intercomponent dynamics to induce wavelike phenomena. The present work offers some insight into how this might be obtained from an engineering of electronic eigenstates to match a structured bath spectral density, highlighting the importance of different energy scales in the problem. We also note that another type of quantum simulator for light harvesting using trapped ions has also recently been demonstrated [64].

Specifically, we use HEOM to prove that although dissipative "population-to-coherence" processes are nonsecular in nature, these often neglected transitions in the perturbative Redfield theory can, following decay of the high-energy state, generate long-lasting coherence between the lower-energy eigenstates. We demonstrate that these quantum beats are a manifestation of real-space coherent motions that could be detectable in a superconducting circuit experiment. To make further connections to general experimental conditions, we also show that interactions with both quantum and classically stochastic fluctuating environments can generate these coherent dynamics, and find that there is an optimal coupling for coherence generation that lies in an intermediate-coupling regime. The possible role of non-Markovianity in these phenomena is also studied, as the structured spectral density we consider has a longer correlation time than the incoherent decay dynamics. However, while we explicitly demonstrate that the use of HEOM is essential to account for the strong 
environmental memory effects (especially for classical noise), the correlation between the generation of coherence and the formal measure of non-Markovianity we use appears to be weak.

The paper is organized as follows. Section II describes the three-state model and the system-bath interaction. Qualitative predictions and concepts emerging from the Redfield approach are given in Sec. III with a presentation of the operational HEOM equations. Section IV presents our numerical results for a quantum or classical noise, and Sec. V provides some discussion and perspectives for future investigations.

\section{MODELS AND PARAMETERS}

\section{A. Electronic system}

A virtue of the model "excitonic" Hamiltonian implemented in Ref. [31] is that its dynamics, with typical timescales of $\mu \mathrm{s}$ in superconducting circuits, remains unchanged when all energetic parameters are rescaled to optical frequencies (factor $\approx 10^{5}$ ). In light of this and in the interest of understanding coherence generation in molecular systems, we will retain the relative energy level and coupling structure of the qubits in Ref. [31], but work at the time and frequency scales of molecular optics and replace qubits with "sites" representing chromophores.

The "electronic" system consists of three chromophoric sites (two-level systems), but the active Hilbert space is confined to the single excitation sector and therefore is described by three states $|n\rangle(n=1,2,3)$ corresponding to a single localized excitation on site $n$. Following the qubit topology of Fig. 1, the model Hamiltonian for the chromophore system is given by

$$
H_{S}=\sum_{n=1}^{3} \varepsilon_{n}|n\rangle\left\langle n\left|+\sum_{n=1}^{3} \sum_{m \neq n=1}^{3} J_{n m}\right| n\right\rangle\langle m| .
$$

As in the experiment, the first two states $(|1\rangle$ and $|2\rangle)$ are tuned to degeneracy $\left(\epsilon_{1}=\epsilon_{2}=0\right)$ and strongly coupled by a coherent coupling $J_{12}$, while state $|2\rangle$ is weakly coupled to a third state $|3\rangle$, through $J_{23}=J_{12} / 10 . J_{13}=0$, which is an approximation very close to the experimental realization [qubit 1 and qubit 3 are not physically close to each other; see Fig. 1(a)]. The energy gap between the degenerate levels and the lower state energy is equal to the first coupling $\epsilon_{3}=-J_{12}$. Diagonalizing this simple Hamiltonian then leads to the eigenstate spectrum shown in Fig. 1(c), which is characterized by a single high-energy state and a low-lying doublet of states with an energy splitting $\approx 2 J_{12}$, approximately ten times smaller than the mean doublet-to-high-energy state gap. Due to this tuning of states, the eigenstates are highly delocalized over the sites. The high-energy "bright" state is approximately given by $|B\rangle=\frac{1}{\sqrt{2}}(|1\rangle+|2\rangle)$, whereas the lower-energy "dark" states are given by $\left|D_{ \pm}\right\rangle=\frac{1}{2}(|1\rangle-|2\rangle) \pm \frac{1}{\sqrt{2}}|3\rangle$.

We remark that here, bright and dark refer to the coupling of these states to the transmission line. As seen in Fig. 1(a), sites 1 and 2 are close to the waveguide and both couple to the excitation field with the same strength. Consequently, the transition dipoles of these sites interfere constructively in the $|B\rangle$ eigenstate, making this bright, while destructive interfer- ence decouples the two dark states $\left|D_{ \pm}\right\rangle$from the excitation fields. Experimentally, this configuration is very useful as it allows optical population of a single, well-defined state from which transport then proceeds, while the nonemissive nature of the dark states prevents radiative losses and noise which might obscure the signatures of energy flow across the site network. Beyond practical considerations, it has also been proposed that using such dark states to reduce radiative losses could boost the efficiency of "bioinspired" organic photovoltaics devices $[18,39,65-70]$. Finally, the resonator is only coupled to state $|3\rangle$, so only $\left|D_{ \pm}\right\rangle$will emit into this channel. Experimentally, it is this resonator emission that is used to quantify the energy transfer from the $|B\rangle$ state. For clarity of discussion, we will not explicitly model the coupling of the system to the excitation and readout fields, but instead consider dynamics beginning with a population prepared in the $|B\rangle$ state.

\section{B. System-bath interaction: Quantum and classically stochastic environments}

In the quantum simulator, noise is generated by applying a stochastic signal along the flux lines that are used to tune the excited-state energies of the individual qubits. This effectively introduces site-selective, stochastic (Gaussian) noise that is diagonal in the basis of the localized qubit excitations (pure dephasing noise), but this noise is essentially classical in nature (vide infra and see Sec. IV B). Nevertheless, this noise is generated by a signal generator that can produce almost arbitrary stochastic power spectra (see Sec. III A), providing a versatile tool for probing dissipative quantum transport. In order to connect with molecular systems, we will consider both classical and quantum noise within a common framework in which the environment is treated as a continuum of quantum harmonic oscillators coupled linearly to the electronic system. Following Ref. [31], we will consider the case of noise applied to only one site of the network, i.e., site 2 , so that the quantum system-bath coupling is given by

$$
H_{S B}=S X=-|2\rangle\langle 2| \frac{1}{\sqrt{2}} \sum_{k} g_{k}\left(a_{k}+a_{k}^{\dagger}\right),
$$

where $S=|2\rangle\langle 2|$ is the system operator and the displacement environmental operator is defined by $X=$ $-\frac{1}{\sqrt{2}} \sum_{k} g_{k}\left(a_{k}+a_{k}^{\dagger}\right)$, where $a_{k}^{\dagger}, a_{k}$ are the bosonic creation and annihilation operators, respectively, of an oscillator of frequency $\omega_{k}$ that is coupled to state $|2\rangle$ with amplitude $g_{k}$. The Hamiltonian of the oscillator bath is $H_{B}=\sum_{k} \omega_{k} a_{k}^{\dagger} a_{k}$ (with $\hbar=1$ ). The system-bath coupling also leads to an energy shift $\lambda=-1 / 2 \sum_{k} g_{k}^{2} / \omega_{k}$ of state $|2\rangle$ (the reorganization energy) that is added to the system Hamiltonian to define an effective system Hamiltonian, $H_{S \text {, eff }}=H_{S}+|2\rangle\langle 2| \lambda$. The total systemenvironment Hamiltonian becomes $H=H_{S \text {, eff }}+H_{S B}+H_{B}$. By diagonalizing $H_{S \text {, eff }}$, all the eigenstates are coupled through the environment by off-diagonal terms of the system coupling operator in the eigenbasis set,

$$
V_{\lambda}=U_{\lambda}^{-1} S U_{\lambda}
$$

The reorganization energy is an indicator of the coupling strength since it modifies the system coupling operator and the energy gap among the eigenstates. We shall use a 


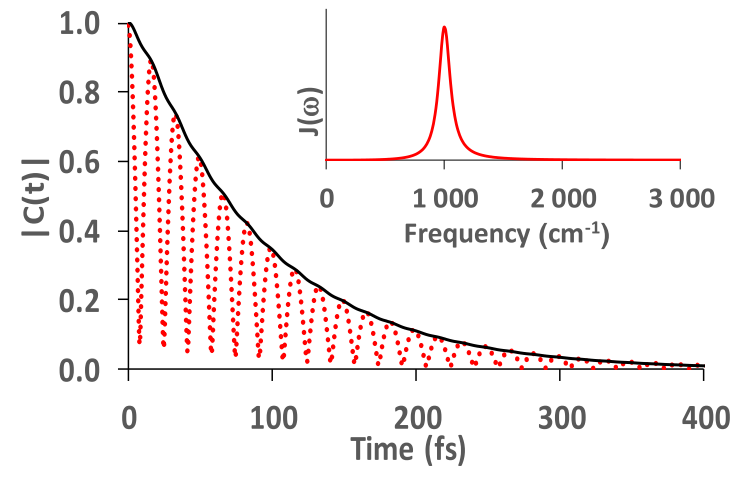

FIG. 2. Modulus of the normalized two-time bath correlation function $C(t-\tau)$ in a.u. with $\tau=0$ at room temperature (solid line) and in the high-temperature limit used to simulate classical noise where the correlation function becomes a real oscillatory function (dots). Inset: Corresponding spectral density in arbitrary units. The system-bath coupling strength and therefore the renormalization energy or the $\eta$ parameter are scanned by varying the $p$ factor in Eq. (6).

dimensionless parameter,

$$
\eta=\lambda / E_{B D_{+}},
$$

giving the ratio between the renormalization energy and the dissipation-free $E_{B D_{+}}$energy gap [55].

Prior to excitation of state $|B\rangle$, we will always assume the environment oscillators are in thermal equilibrium with respect to their free Hamiltonian $H_{B}$. With this common assumption, the behavior of the reduced density matrix of the electronic subsystem is completely characterized by the environment's spectral function $J(\omega)=\sum_{k}\left(g_{k}^{2} / \omega_{k}\right) \delta\left(\omega-\omega_{k}\right)$ and its temperature through the Bose function $n(\omega)=\left(e^{\beta \omega}-1\right)^{-1}$, where $\beta=1 / k_{B} T$ and $k_{B}$ is the Boltzmann constant. Both appear in the thermal two-time correlation function of the oscillator displacements, which ultimately determines the dissipative physics of the system (see below) [71]. The correlation function is given by $C(t-\tau)=\operatorname{Tr}_{B}\left[\rho_{B} X(t) X(\tau)\right]$, where $\rho_{B}$ is the equilibrium density matrix of the environment oscillators and the time-dependent operators are in the Heisenberg picture with respect to the Hamiltonian $H_{B}$ of the environmental displacement operator $X$. This leads to

$$
C(t-\tau)=\frac{1}{\pi} \int_{-\infty}^{+\infty} d \omega J(\omega) n(\omega) e^{i \omega(t-\tau)} .
$$

In the context of open system theory, the difference between quantum and classical stochastic noise is most clearly seen in $C(t)$; the two-time correlation function for noncommuting operators is complex valued, while for a classical scalar variable, it is real. From Eq. (5) and the fact that $J(-\omega)=-J(\omega)$, it can be seen that $C(t)$ becomes real in the limit of $\beta \rightarrow 0$ (hightemperature limit) and we shall later exploit this to extract results about classical noise from our HEOM simulations. This is further illustrated in Fig. 2 for the structured spectral density that we will consider in our numerical results. The spectral density here is a superohmic Lorentzian function,

$$
J(\omega)=\frac{p \omega^{3}}{\Lambda_{1}\left(\Omega_{1}, \Gamma_{1}\right) \Lambda_{2}\left(\Omega_{2}, \Gamma_{2}\right)},
$$

where $\Lambda_{k}=\left[\left(\omega+\Omega_{k}\right)^{2}+\Gamma_{k}^{2}\right]\left[\left(\omega-\Omega_{k}\right)^{2}+\Gamma_{k}^{2}\right]$.

The parameters are chosen to create a sharp spectral density peaked at the energy gap $E_{B D}$, as shown in the inset of Fig. 2 . The numerical values of the parametrization used are given in Appendix B, with the $p$ parameter being used as a scaling factor that allows us to vary the reorganization energy of the bath.

\section{SIMULATION TECHNIQUES FOR OBTAINING AND CHARACTERIZING REDUCED DENSITY MATRICES}

\section{A. Redfield equations}

For open quantum systems, the principal object of interest is the reduced density matrix of the system $\rho_{S}(t)=$ $\operatorname{Tr}_{B}[\rho(t)]$, where $\rho(t)$ is the joint density matrix of the systemenvironment state. In general, determining $\rho(t)$ in order to obtain $\rho_{S}(t)$ is highly demanding; however, it is possible in many cases to find approximations that greatly simplify this task and which also provide very useful concepts and intuition for discussing more complex open physics.

For the case of weak coupling (second-order perturbation theory with respect to system-bath coupling) leading to a broad spectral function characterized by a cutoff frequency $\omega_{c}$ that is much larger than any energy difference in the system Hamiltonian $H_{S \text {,eff }}$, the dynamics of $\rho_{S}(t)$ are often well described by the Bloch-Redfield theory. Detailed derivations of the BlochRedfield master equation can be found in Refs. [71,72]; here we shall simply state the results of immediate consequence for our results and discussion. Following the Born-Markov approximation, the equation of motion for the reduced density matrix in the interaction picture, $\tilde{\rho}_{S}(t)=e^{-i H_{S, \text { eff }} t} \rho_{S}(t) e^{+i H_{S, \text { eff }} t}$, is given by the time-local master equation

$$
\dot{\tilde{\rho}}_{n m}(t)=\sum_{j, k} R_{n m j k} \tilde{\rho}_{j k}(t) e^{i\left(E_{n m}-E_{j k}\right) t},
$$

where $\rho_{n m}=\left\langle E_{n}|\rho| E_{m}\right\rangle$, and $\left|E_{m}\right\rangle$ is the $m$ th eigenstate of $H_{S \text {,eff }}$ with energy $E_{m}$, which we number in order of ascending energy. This form, containing explicit time dependence, is often referred to as the nonsecular Bloch-Redfield equation, distinguishing it from the secular Bloch-Redfield equations, which are obtained from Eq. (7) by only retaining terms in the right-hand side summation for which $E_{n m}-E_{j k}=0$, where $E_{n m}=E_{n}-E_{m}$. This last approximation is normally justified when the energy differences between different transition energies, $E_{n m}-E_{j k} \gg R_{n n j k}^{-1}, \forall n, m, i, j$, so that on the typical timescales on which the density matrix evolves under the action of the Redfield tensor $R_{n m j k}$, the highly oscillatory terms with $E_{n m}-E_{j k} \neq 0$ average to zero. When this is indeed valid, the secular Bloch-Redfield equations have a particularly simple form, as population (diagonal) and coherence (off-diagonal) terms of $\rho_{S}$ are completely decoupled. The populations then obey a Pauli (kinetic) master equation, while any coherences present in the initial condition independently and exponentially decay to zero. Crucially, there are no terms in the secular Bloch-Redfield (SBR) equations that allow for the ex nihilo generation of coherences.

However, nonsecular terms can create dynamical coupling between populations and coherences, as has been widely discussed in the context of ultrafast spectroscopies. The 
significance of these terms has also recently been highlighted in a number of papers, showing that their inclusion often provides a more accurate description when compared with more advanced numerical treatments of open quantum systems [73]. Interestingly, well-known problems related to the potential lack of positivity of reduced density matrices under BR evolution have also been shown to arise from problems related to the failure of the Born-Markov assumption, rather than the structure of the master equations when nonsecular terms are included [74].

\section{B. Nonsecular generation of spontaneous coherence via incoherent decay}

Of particular relevance for our three-level model is the structure of the population-to-coherence elements of the Redfield tensor $R_{n m i i}=R_{m n i i}^{*}$, especially the term $R_{D_{+} D_{-} B B}$, which describes the generation of coherence by a population in the initially excited state $|B\rangle$. This is given by

$$
\begin{aligned}
R_{D_{+} D_{-} B B}= & \pi V_{D_{+} B}^{*} V_{D_{-} B} J\left(E_{B D_{+}}\right)\left[n\left(E_{B D_{+}}\right)+1\right] \\
& +\pi V_{D_{+} B}^{*} V_{D_{-} B} J\left(E_{B D_{-}}\right)\left[n\left(E_{B D_{-}}\right)+1\right] .
\end{aligned}
$$

Comparing this to the incoherent decay rate from state $|B\rangle$ to the lower-energy doublet of states (population-to-population transfer),

$$
\begin{gathered}
\left.R_{D_{+} D_{+} B B}=2 \pi\left|V_{B D_{+}}\right|^{2} J\left(E_{B D_{+}}\right)\left[n\left(E_{B D_{+}}\right)+1\right)\right], \\
\left.R_{D_{-} D_{-} B B}=2 \pi\left|V_{B D_{-}}\right|^{2} J\left(E_{B D_{-}}\right)\left[n\left(E_{B D_{-}}\right)+1\right)\right],
\end{gathered}
$$

we can see that if $J\left(E_{D_{ \pm} B}\right), n\left(E_{B D_{ \pm}}\right)$and matrix elements $V_{D_{ \pm} B}$ are equal, then $R_{D_{+} D_{+} B B}=R_{D_{-} D_{-} B B}=R_{D_{+} D_{-} B B}$. For this case, and in the absence of the time dependence associated with the term $R_{D_{+} D_{-} B B}$ in Eq. (7), the incoherent decay of state $B$ would create population and coherence in the lower doublet at an equal rate, i.e., it would tend to generate a coherent superposition of the lower doublet states. Indeed, if the decay of the $|B\rangle$ state is much faster than the oscillation period (set by $E_{D_{+} D_{-}}$), one would expect the relaxation to occur into a pure superposition state of $|\psi\rangle \approx\left|D_{+}\right\rangle \pm\left|D_{-}\right\rangle$(with phase depending on the sign of the matrix elements). If the decay is much slower than the oscillatory period, the coherence generation will be negligible, leading to an incoherent mixture of (approximately equal) populations in the doublets.

Within the scope of BR theory, the eigenstate level scheme and spectral density that we consider enables the conditions for coherence generation to be almost perfectly met, at least for small reorganization energy, when the model parameters remain close to those predicted by the eigenstates of $H_{S}$. First, the matrix elements for the transition between the $|B\rangle$ state and the doublets $\left|D_{ \pm}\right\rangle$induced by the spatially local operator $V_{\lambda}$ (3) are almost equal due to the real-space delocalization of the states and the large energy gap between the $|B\rangle$ and $\left|D_{ \pm}\right\rangle$manifolds (their fractional difference is no more than $\left.\approx J_{23} / J_{12} \ll 1\right)$. Second, by applying a spectral function that is symmetric and peaked at an energy $\frac{1}{2}\left(E_{B D_{+}}+E_{B D_{-}}\right)$, we obtain equality of $J\left(E_{D_{ \pm} B}\right)$. Third, by working at a temperature such that $\beta E_{B D_{ \pm}} \gg 1$ or $\beta E_{B D_{ \pm}} \ll 1$, all relevant elements of the Redfield tensor approach equality. This only leaves the condition that the transition rates for decay of the $|B\rangle$ state should be faster than the period of coherent oscillations in the $\left|D_{ \pm}\right\rangle$states. This can be controlled by varying the coupling strength to the environment, although we note that obtaining this condition violates the standard application of the Markov approximation, additionally motivating our use of nonperturbative HEOM methods (see below).

Finally, we note that the same qualitative analysis can be applied to coherences generated between the $|B\rangle$ and $\left|D_{ \pm}\right\rangle$ states due to decay of the $|B\rangle$ state. However, the relevant oscillatory time period to compare to the decay rate is now set by the bright-dark state splitting $E_{B D}$, which is ten times larger than $E_{D_{+} D_{-}}$. It could therefore be possible to find a parameter space in which large interdoublet coherence is generated without any significant coherence generation between the bright and dark states. This scenario defines what we mean by coherence generation arising from incoherent relaxation.

\section{HEOM}

We recall here the derivation already presented in previous works [54,56-61] and give, for the purpose of completeness, the main equations. For the efficiency of the HEOM algorithm, the spectral density is parametrized so that the two-time bath correlation function is expressed as a sum of complex exponential functions [75],

$$
C(t-\tau)=\sum_{k=1}^{n_{\text {cor }}} \alpha_{k} e^{i \gamma_{k}(t-\tau)} .
$$

Explicit expressions of the $\alpha_{k}$ and $\gamma_{k}$ by the analytical integration of Eq. (5) with the superohmic parametrization of the spectral density (6) can be found in the appendix of Ref. [76]. $n_{\text {cor }}$ is the sum of the four terms coming from the four simple poles in the upper complex plane and, in principle, an infinite number of terms related to the poles (Matsubara frequencies) of the Bose function on the imaginary axis $v_{j}$. In practice, we find that the number of Matsubara terms remains small (about 10) at and above room temperature for the model under study. The complex conjugate of the correlation function can be expressed by keeping the same coefficients $\gamma_{k}$ in the exponential functions with modified coefficients $\tilde{\alpha}_{k}$ according to

$$
C^{*}(t-\tau)=\sum_{k=1}^{n_{\text {cor }}} \tilde{\alpha}_{k} e^{i \gamma_{k}(t-\tau)},
$$

with $\tilde{\alpha}_{1}=\alpha_{2}^{*}, \tilde{\alpha}_{2}=\alpha_{1}^{*}, \tilde{\alpha}_{3}=\alpha_{4}^{*}, \tilde{\alpha}_{4}=\alpha_{3}^{*}$, and $\tilde{\alpha}_{j \text {,matsu }}=$ $\alpha_{j \text {,matsu }}$, where the $\alpha_{m}$ with $m=1,4$ are related to the four poles of the superohmic Lorentzian function and $\alpha_{j \text {,matsu }}$ refer to the Matsubara terms [77].

By assuming an initial factorization of the total density matrix, the time evolution of the reduced density matrix, in interaction representation $\tilde{\rho}_{S}(t)$, is given by

$$
\begin{aligned}
\tilde{\rho}_{S}(t) & =\operatorname{Tr}_{B}\left[e^{\int_{0}^{t} d \tau L(\tau)} \rho_{B}^{e q} \tilde{\rho}_{S}(0)\right] \\
& =e^{\int_{0}^{t} d \tau \int_{0}^{\tau} d t^{\prime} \operatorname{Tr}_{B}\left[L(\tau) L\left(t^{\prime}\right) \rho_{B}^{e q}\right]} \tilde{\rho}_{S}(0),
\end{aligned}
$$

where $L(t)$. $=-\frac{i}{\hbar}[S(t) X(t),$.$] is the Liouvillian of the system-$ bath interaction with the system coupling operator in interaction representation, $S(t)=e^{i \hat{H}_{S, \text { eff }} t} \hat{S} e^{-i \hat{H}_{S, \text { eff }} t}$, and the bath operator as given above. Expressions (11) and (12) for $C(t-\tau)$ and $C^{*}(t-\tau)$ correspond to the relaxation of $n_{\text {cor }}$ effective 
bath modes. Each set of the corresponding occupation numbers is represented by a collective index $\mathbf{n}=\left\{n_{1}, \ldots, n_{n_{\mathrm{cor}}}\right\}$ and is associated to an auxiliary density matrix. The master equation is then written as a time-local hierarchical system of coupled equations among the auxiliary operators. Each matrix can communicate only with the superior and inferior level in the hierarchy for which one occupation number is varied by one unity, $\mathbf{n}_{k}^{ \pm}=\left\{n_{1}, \ldots, n_{k} \pm, \ldots, n_{n_{\text {cor }}}\right\}$,

$$
\begin{aligned}
\dot{\rho}_{\mathbf{n}}(t)= & i \sum_{k=1}^{n_{\text {cor }}} n_{k} \gamma_{k} \rho_{\mathbf{n}}(t)-i\left[S(t), \sum_{k=1}^{n_{\text {cor }}} \rho_{\mathbf{n}_{k}^{+}}(t)\right] \\
& -i \sum_{k=1}^{n_{\text {cor }}} n_{k}\left[\alpha_{k} S(t) \rho_{\mathbf{n}_{k}^{-}}-\tilde{\alpha}_{k} \rho_{\mathbf{n}_{k}^{-}} S(t)\right] .
\end{aligned}
$$

In this hierarchy of auxiliary density matrices, the system density matrix is given by the top row, i.e., for $\mathbf{n}=\{0, \ldots, 0\}$, hence $\tilde{\rho}_{S}(t)=\rho_{\{0, \ldots, 0\}}(t)$. The level of the hierarchy is chosen until convergence is reached for the system density matrix. As previously stated, it can be seen that the equations of motion that determine the reduced density matrix of the system are completely determined by the expansion coefficients of the correlation functions $C(t)$ and $C^{*}(t)$ that appear in Eqs. (11) and (12), and which are ultimately determined by the environment spectral function and temperature.

The HEOM equations are efficient to go beyond the second-order perturbative regime of the Redfield equations even if the Markovian approximation could still be valid at higher orders. However, stronger couplings are often linked to non-Markovian dynamics. The signature of non-Markovian behavior for strong system-bath coupling is analyzed in Appendix A, where we also illustrate convergence of HEOM equations and compare numerically exact HEOM simulations with Redfield results.

\section{RESULTS}

\section{A. Quantum noise}

In each simulation, the initial state is the bright eigenstate $|B\rangle$ and the bath is at room temperature, $T=298 \mathrm{~K}$, which corresponds to quantum noise for our spectral density, as the peak frequency $\Omega \approx 5 k_{B} T$. We note, however, that this temperature gives an energy scale $k_{B} T / E_{D_{+} D_{-}} \approx 3$, which would be expected to drive equal (mixed) populations of the doublet population and rapid coherence loss. From the analysis based on the Redfield theory, we first examine the evolution of the main tensor elements to predict the best range of the $\eta$ parameter [Eq. (4)] to create the expected long-lived superposed state in the doublet. The three main tensor elements of the "downhill" transitions at $T=298 \mathrm{~K}$, $R_{D_{+} D_{+} B B}, R_{D_{-} D_{-} B B}, R_{D_{+} D_{-} B B}$, are displayed in Fig. 3 as a function of the $\eta$ parameter by accounting for the variation of the coupling $V_{\lambda}$ [Eq. (3)] and of the eigenenergy gap induced by the renormalization energy. The best expected domain for the coherence creation appears to be around $\eta=0.015$, which corresponds here to a renormalization of about $15 \mathrm{~cm}^{-1}$. This value provides the largest decay rate while maintaining equality of the three relevant Redfield tensor terms. The reverse rates, $R_{B B D_{-} D_{-}}, R_{B B D_{+} D_{-}}$, remain negligible at room temperature due to detailed balance, potentially extending the lifetime of any decay-generated coherent states. Simulation with HEOM

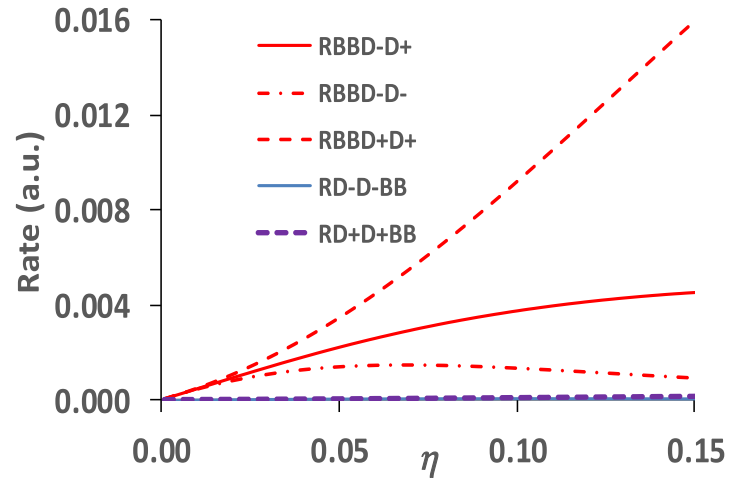

FIG. 3. Main Redfield tensor elements for population-topopulation or population-to-coherence transfer as a function of the coupling $\eta$ parameter in Eq. (4).

will allow us to probe stronger couplings beyond the perturbative regime and examine the stability of the process.

Figure 4 shows the population evolution [i.e., the diagonal elements of $\left.\rho_{S}(t)\right]$ in the $|B\rangle$ state (solid lines) and in the doublet $\left|D_{+}\right\rangle$(dashed lines) and $\left|D_{-}\right\rangle$(dotted lines) for different coupling regimes. In the perturbative regime [Fig. 4(a)], the decay is monotonous while for the strongcoupling case [Fig. 4(b)], oscillations occur which are related to
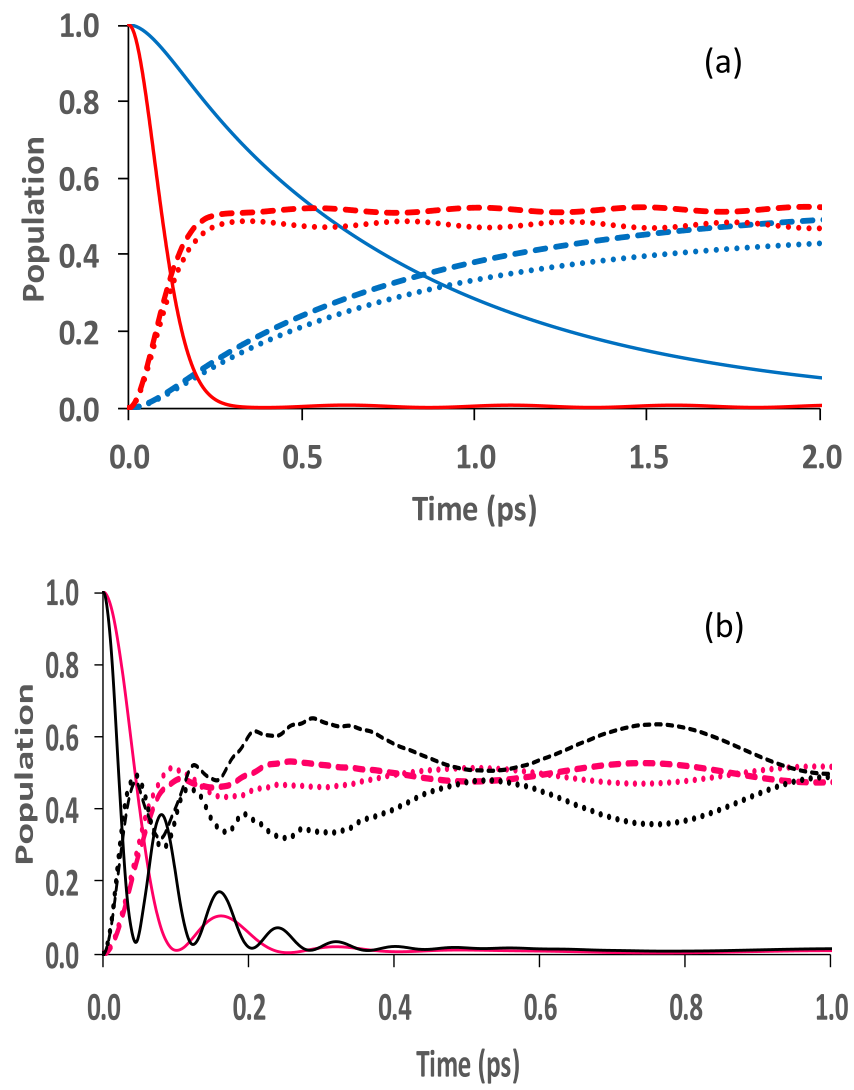

FIG. 4. Population evolution of the bright state $|B\rangle$ (solid lines) and of the dark doublet $\left|D_{+}\right\rangle$(dotted lines) and $\left|D_{-}\right\rangle$(dashed lines) for different coupling strengths. (a) Weak system-bath coupling $\eta=$ 0.001 (blue) and $\eta=0.01$ (red); (b) strong coupling $\eta=0.04$ (pink) and $\eta=0.16$ (black). 


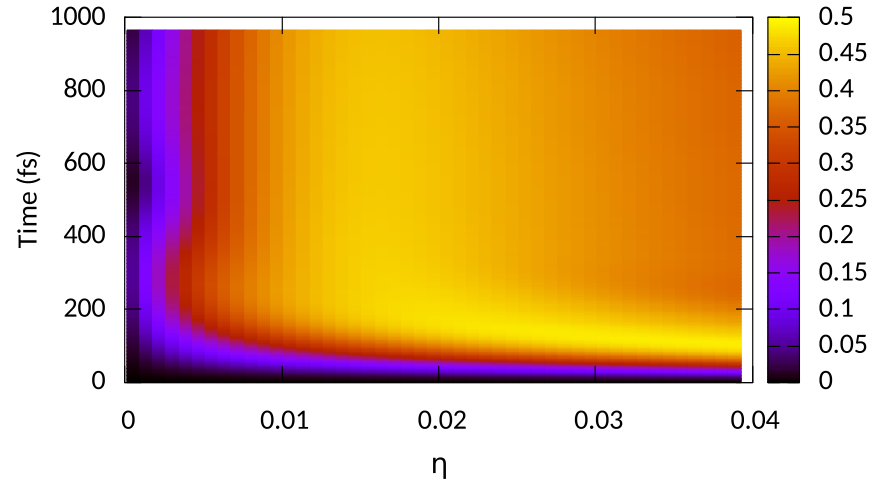

FIG. 5. Isovalue contours in the modulus of the coherence between the doublet states $\rho_{D_{+} D_{-}}(t)$ for different $\eta$ parameters [Eq. (4)] at $T=298 \mathrm{~K}$.

quasireversible energy exchange between the system and the environment, as the coupling strength exceeds the linewidth of the spectral function (strong-coupling cavity limit). These latter dynamics lead to features in the measure of nonMarkovianity that we present in Appendix A, as predicted in Ref. [78]. The possible creation of a superposition state is suggested by the simultaneous growing of population in the $\left|D_{ \pm}\right\rangle$doublet states, although this could also arise without generating any coherence in the doublet. Close to the best expected coupling regime for $\eta=0.01$ (red curves), the populations rise monotonically and plateau to equilibrium values expected from the Boltzmann distribution at this temperature. The populations
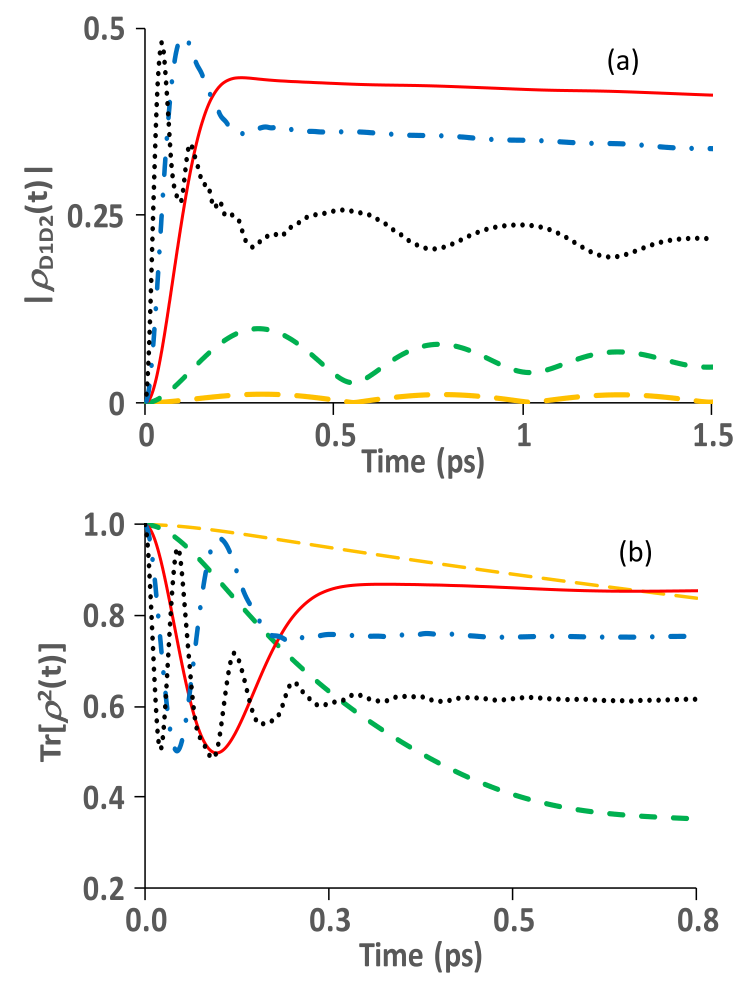

FIG. 6. (a) Modulus of the coherence between the doublet states $\rho_{D_{+} D_{-}}(t)$ for different $\eta$ parameters. (b) Purity of the system matrix density $\operatorname{Tr}\left[\rho_{S}^{2}(t)\right]$ for the same $\eta$. Dotted line: $\eta=0.16$; dash-dotted line: $\eta=0.04$; solid line: $\eta=0.01$; short-dashed line: $\eta=10^{-3}$; long-dashed line: $\eta=10^{-4}$.
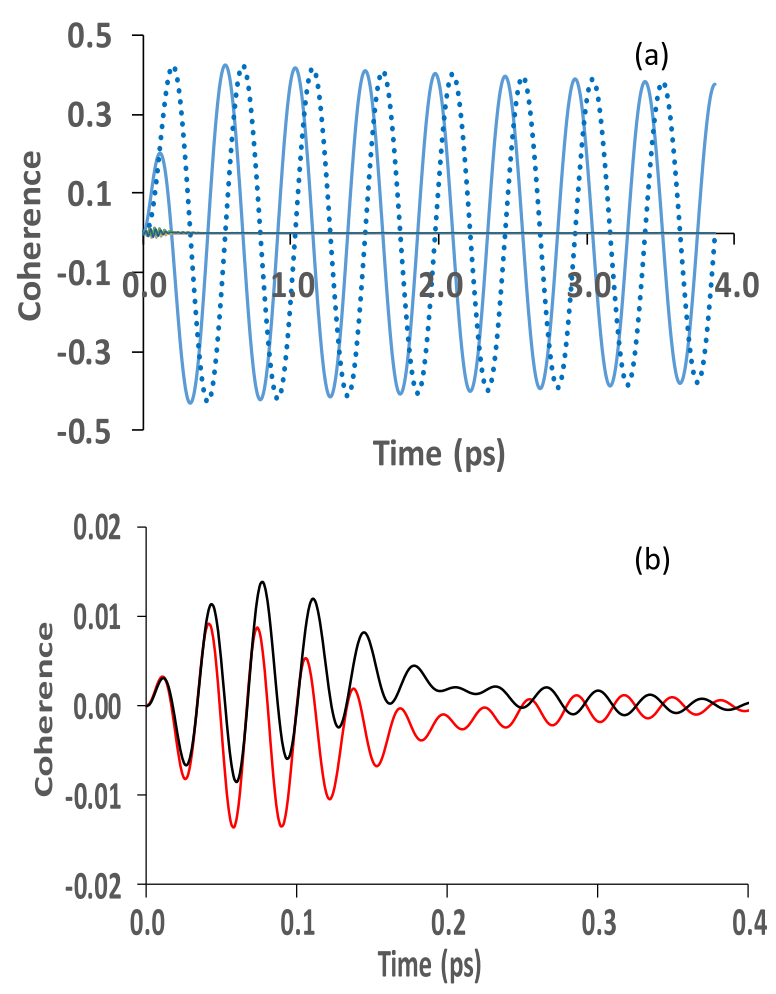

FIG. 7. (a) Coherence $\rho_{D_{+} D_{-}}(t)$ (solid blue line: real part; blue dashed line: imaginary part) for $\eta=0.01$. All the other coherences are in black lines. (b) Zoom of the real part of the coherences $\rho_{B D_{-}}(t)$ (red line) and $\rho_{B D_{+}}(t)$ (black line).

of the two doublet states show oscillatory behavior in the strong regime $\eta=0.16$ (black curves), with a period and duration much longer than the oscillations seen in the decay of the bright state. We will return to this nonperturbative effect in Sec. IV B.

The critical observable, i.e., the modulus of the coherence $\left|\rho_{D_{+} D_{-}}(t)\right|$ between the doublet states, may be seen in Fig. 5 as contour plots in a time and $\eta$ parameter map or in Fig. 6(a) for some selected couplings. The amplitude of the created coherence shows a clear dependence on the system-bath coupling. In the optimal situation corresponding to values near $\eta=0.015$, as predicted by the Redfield analysis, the coherence modulus reaches very close to the maximum possible value of 0.5 in about $100 \mathrm{fs}$ and remains stable for more than 1 ps. For very weak coupling, a coherence is observed but its amplitude remains below 0.1 . For the strong coupling, the early dynamics leads to a high amplitude around 0.5 , but due to the bath interaction the asymptotic value stabilizes below the optimal coherence.

The purity of the system density matrix $\operatorname{Tr}\left[\rho_{S}^{2}(t)\right]$ is given in Fig. 6(b). In the weak-coupling cases $\left(\eta=10^{-4}\right.$ or $\left.10^{-3}\right)$, the purity is mainly determined by the mixed state with the initial state which is not yet relaxed. The purity confirms that the most favorable situation is the moderate-coupling case around $\eta=0.01$, where the asymptotic purity is well above the purity of a Boltzmann mixture at room temperature $(\approx 0.5)$ and shows that incoherent relaxation produces a superposition state in the doublet with relatively little entropy generation.

Figure 7(a) illustrates the stability of the created coherence $\rho_{D_{+} D_{-}}(t)$ for a favorable case with $\eta=0.01$ and the difference 


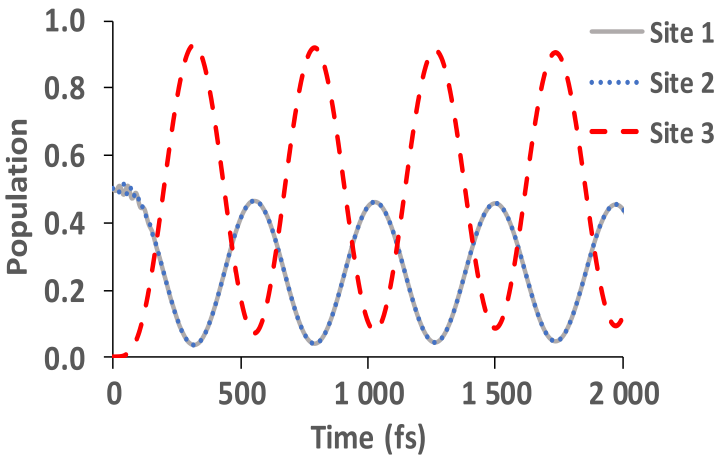

FIG. 8. Populations in the real-space site basis of the coupled network for coupling to quantum noise $(\eta=0.01)$.

with the other coherences $\rho_{B D_{-}}(t)$ or $\rho_{B D_{+}}(t)$. As shown in Fig. 7(b), the latter completely disappears after $500 \mathrm{fs}$ and their amplitudes never exceed 0.015 so they are smaller by more than one order of magnitude. As previously discussed, this establishes that the coherence generation arises from an incoherent decay.

These beats could potentially be detected in an experimental setup similar to Ref. [31]. The rapid relaxation effectively prepares a nearly pure superposition state that coherently evolves over a subsequent time $t$ as $|\psi(t)\rangle \approx \frac{1}{\sqrt{2}}\left(e^{-i E_{D_{+}} t}\left|D_{+}\right\rangle+\right.$ $\left.e^{-i E_{D_{-}} t}\left|D_{-}\right\rangle\right)$. Expanding this state in the site basis and noting that $\left|D_{+}\right\rangle+\left|D_{-}\right\rangle=|1\rangle-|2\rangle$ and $\left|D_{+}\right\rangle-\left|D_{-}\right\rangle=|3\rangle$, it can be easily seen that the evolution of the wave-function phases leads to oscillatory real-space motion of the excitation between sites 1,2, and 3. This is illustrated in Fig. 8, where a periodic and near-unity population of site 3 with frequency $E_{D_{+} D_{-}}$can be seen. As the resonator emission only arises from the population of site 3 , oscillations in its population should be observable as a periodic modulation in the resonator signal at a frequency $E_{D_{+} D_{-}}$.

The stability of the coherence creation via the incoherent decay was checked with respect to the shape of the spectral density. We compare the sharp spectral density with a broader one (the parameters are given in the Appendix). They are schematized in the inset of Fig. 9. The renormalization energy is calibrated to be nearly equal in both cases. Figure 9(a) presents the population evolution in the $|B\rangle$ and $\left|D_{ \pm}\right\rangle$doublet for $\eta=0.01$. The decay is slower in the broad peak case, but the population in the two doublet states is still growing simultaneously. As shown in Fig. 9(b), the coherence $\rho_{D_{-} D_{+}}(t)$ presents a similar profile in both coupling schemes. We note that this example serves to show that the coherence generation is a result of the coupling matrix elements and transitions rates (thus appearing at the level of the master equation), and do not arise from vibronic mixing effects that require a strong coupling to a resonant and sharp (underdamped) vibrational mode at the Hamiltonian level. This suggests that the conditions for noise engineering, both in quantum simulators and physical realizations, that are required for noise-driven coherence are, in fact, rather lenient.

While we have shown in this section that it is possible to find a parameter regime where quantum noise can lead to coherence generation via incoherent relaxation, the longevity of the resulting, near-perfect superposition states is perhaps not so
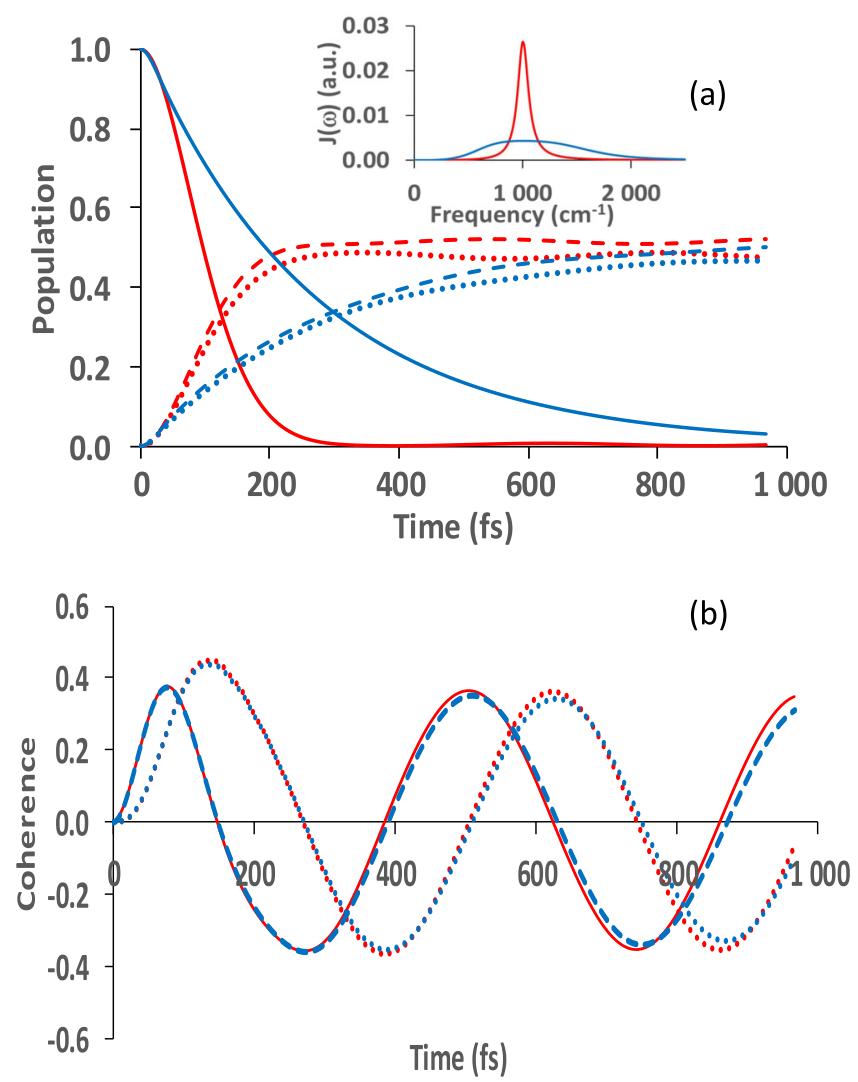

FIG. 9. Comparison of the population evolution and of the coherence $\rho_{D_{-} D_{+}}(t)$ in the doublet states for the two spectral densities shown in the inset. The renormalization energies correspond to $\eta=$ 0.01. Thin peak: red curves; broad peak: blue curves. (a) Population in the bright state $|B\rangle$ (solid line) and the dark states $\left|D_{-}\right\rangle$(dotted line) and $\left|D_{+}\right\rangle$(dashed line). (b) Real part of the coherence $\rho_{D_{-} D_{+}}(t)$ (thin peak: solid line; broad peak: dashed line) and imaginary parts (thin peak: red dots; broad peak: blue dots).

surprising. Due to the absence of incoherent transitions back to the high-energy bright state, the only mechanisms of dephasing in the doublet are due to intradoublet relaxation and/or pure dephasing. For both the peaked and broad spectral densities that we have used, the spectral weight at the energy gap $E_{D_{+} D_{-}}$ is extremely small and pure dephasing vanishes at long times [4,7], making the doublet state effectively decoupled from the environment. As we shall show, this situation changes dramatically in the experimentally relevant case of classical noise.

\section{B. Classical stochastic noise}

At the limit of very high temperature, the bath correlation function becomes real and therefore corresponds to a colored classically stochastic noise (see Fig. 2). This suggests that it should be possible to simulate the effects of stochastic noise, including any non-Markovianity, by making the simulation "temperature" much larger than the other intrinsic energy scales of the system and bath, while rescaling the coupling to the bath to maintain physically reasonable transition rates. In the Golden Rule approximation, the decay rates depend on $J(\omega)[n(\omega)+1]$, which becomes $J(\omega) k_{B} T / \omega$ at the hightemperature limit. In order to keep the transition rates at 
similar values to those in Sec. IV A (which also maintains the significance of our parameter $\eta$ ), we set an artificially high temperature $\left(10^{3} \mathrm{~K}\right)$ while simultaneously dividing the renormalization energy by a factor of $k_{B} T$. We note that this procedure captures the essential "infinite temperature" property of classically stochastic noise: the up and down transition rates are now effectively equal so that any coherence created by relaxation is now subject to potentially strong dephasing noise arising from the rapid transitions that drive the system towards the maximally mixed Boltzmann state.

Figure 10(a) presents the population evolution for different $\eta$ parameters. The case $\eta=0.01$ (red curves) may be compared with the quantum noise case (see Fig. 4) where this coupling range gave optimal generation of the doublet state. With a classical noise case, the superposition is still created on ultrafast timescales, but the peak coherence amplitude is smaller (0.3) and coherence is completely destroyed after just $0.1 \mathrm{ps}$, as seen in Fig. 10(b) (red curves). This dephasing time is much faster than the period of coherent oscillations in the dark doublet, so no beating can be resolved in the time domain. Reducing the coupling by an order of magnitude leads to large-amplitude coherence generation within $\sim 200 \mathrm{fs}$, but the dephasing rate is now slower than the beating period, allowing about 2-3 cycles of beating to be observed over about $1 \mathrm{ps}$. For very weak coupling $\eta=10^{-4}$ (blue curves), the population decay is very
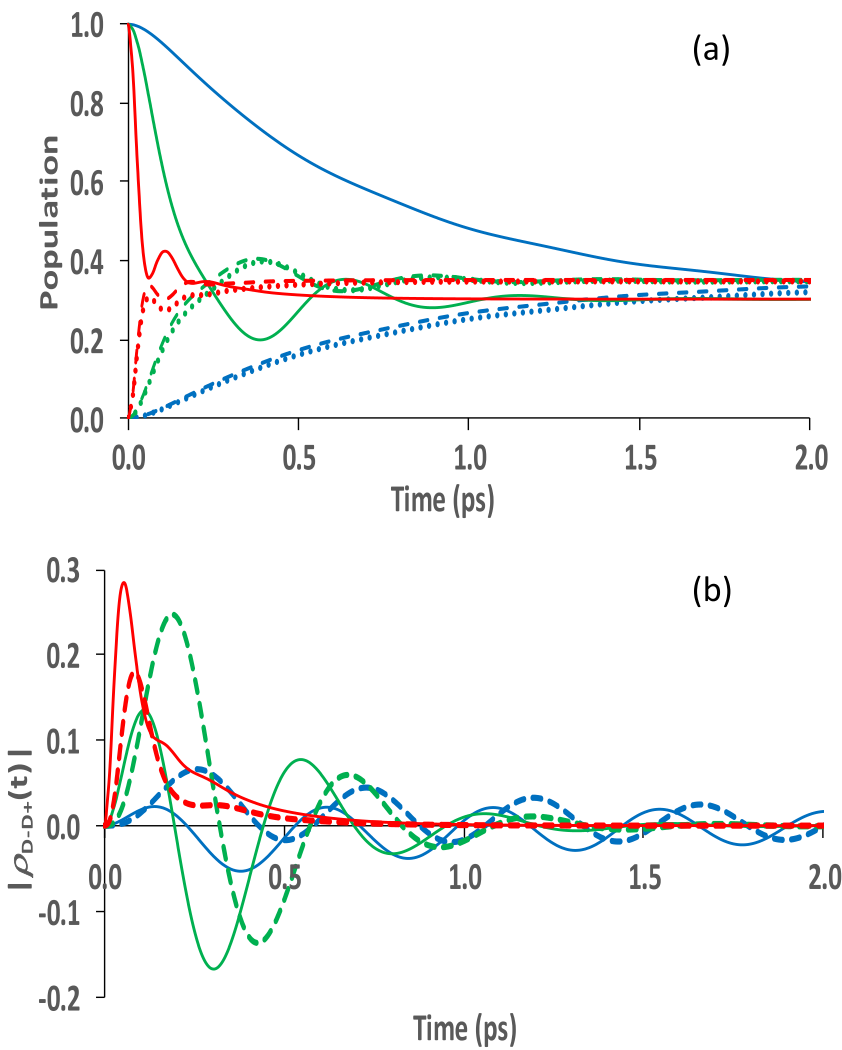

FIG. 10. (a) Population evolution of the bright state $|B\rangle$ (solid lines) and in the dark doublet $\left|D_{-}\right\rangle$(dotted line) and $\left|D_{+}\right\rangle$(dashed line) for three coupling parameters : $\eta=10^{-2}$ (red), $\eta=10^{-3}$ (green), and $\eta=10^{-4}$ (blue). (b) Real part (solid line) and imaginary part (dotted line) of the coherence between the doublet state $\rho_{D_{-} D_{5}}(t)$ for the different parameters $\eta$. slow, but a coherence of weak amplitude $(<0.1)$ is created and maintained during the entire decay (about 2 ps) of the system to thermal equilibrium.

We therefore confirm that the noise-induced generation of coherences survives in the case of classically stochastic noise and, although it is much more fragile, as seen in Fig. 10, there is a parameter regime where it is possible to resolve the quantum oscillations in the temporal domain.

Compared to the quantum (cold) case, we note the following differences in coherence generation that may be relevant for future experiments in superconducting quantum circuits and/or molecular array architectures. First, the near equality of upward and downward transition rates creates a competition between the fast relaxation needed to generate coherence and the dephasing that arises from the uphill transitions. As shown above, in order to resolve the beats, a compromise must be struck between the maximum possible amplitude of the coherence and the lifetime of the oscillations. The optimum point will depend on the method of detection and the leveraging between acceptable signal-to-noise (favoring large amplitude) and the available time and frequency resolution. Second, at larger coupling strengths, oscillations are also seen in the populations of the eigenstates which are damped on the same timescale as the corresponding coherences. Unlike the quantum case, these oscillations are not due to reversible energy exchange with the environment, but result from the coherent real-space motion of the excitation in the doublet states. Again, an approximate but intuitive understanding can be obtained from the structure of the Redfield equations.

Due to the choice of coupling to the environment (local coupling to site 2), the rapid initial nonsecular relaxation in the regime of coherence generation (relaxing to a superposition state) can also be seen as a relaxation of the bright state into the nonstationary dark state $|D\rangle=\frac{1}{\sqrt{2}}\left(\left|D_{+}\right\rangle+\left|D_{-}\right\rangle\right)$. As all the uphill rates are also the same, subsequent relaxation back to the bright state only arises when this nonstationary state is populated. However, due to the coherent evolution of the $|D\rangle$ state, excitations move in real space to site 3, which is not coupled to the environment, so that the population in the doublet states is temporarily unable to make any uphill transitions. As the oscillatory quantum beats return population to the $|D\rangle$ state, the uphill transitions become allowed again, depopulating the dark doublet states in a periodic way. These motion-induced modulations of the uphill transition rate are the origin of the eigenstate population oscillations seen in Fig. 10(a).

Interestingly, this modulation of the transition rates effectively results in a transient and periodic violation of detailed balance $[69,79]$, as the suppression of the upward transitions leads to an "overshoot" of population transfer from the bright state, as if the bath were (temporally) at a much lower temperature. This effect is most prominent for the intermediate coupling $(\eta=0.01)$, where the coherence is both large enough and long-lasting enough to allow a few near-complete oscillations of the excitation between sites 1,2, and 3. This interpretation is confirmed by looking at the populations in the site basis for this coupling, as shown in Fig. 11. Comparing to Fig. 10(a), we see that the eigenstate oscillations occur at precisely the times when site 3 is maximally populated and uphill transitions are 


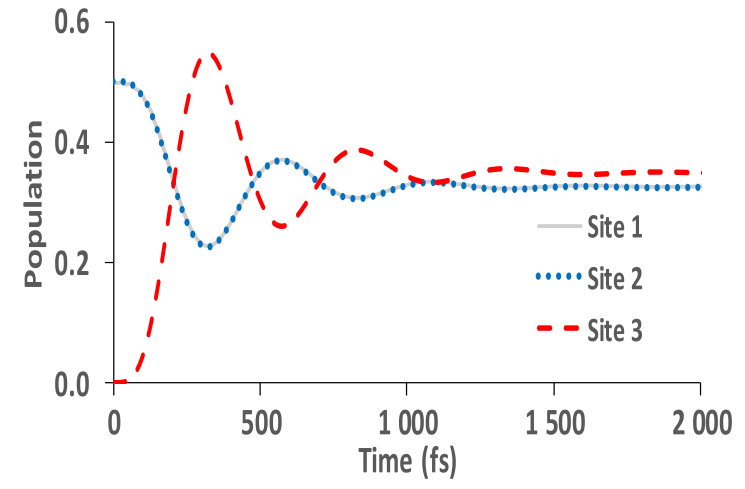

FIG. 11. Populations in the real-space site basis of the coupled network for coupling to classical noise $(\eta=0.001)$.

suppressed. Experimentally, these coherent modulations of the bright state decay would be detectable through the emission in the transmission and excitation waveguide and would be in antiphase with modulations in the resonator signal. Indeed, because these coherent dynamics directly affect the eigenstate populations, it is likely that they will be even easier to detect for the oscillations in the eigenstate coherences that appear in the regime quantum dissipation. We note that this effect is also responsible for the oscillations in the modulus of the coherence that can be seen in Fig. 12.

For completeness, we finally comment on the nature of the population oscillations in the quantum case of Fig. 4. These arise from a completely different physics, and the oscillations only occur between the doublet states at strong coupling. This is due to the increasing relevance of the reorganization energy, compared to the electronic couplings and detunings between the sites of the network. Again, due to the coupling of the bath to just site 2, this has the effect of detuning that site so that the effective eigenstates in the lower doublet no longer correspond to the fully delocalized states $\left|D_{+}\right\rangle$and $\left|D_{-}\right\rangle$. The increasing amplitude of the oscillations that we observe with stronger coupling are essentially related to the increasing misalignment of the measurement and effective eigenstate basis. It is clear that a nonstationary state of these eigenstates is still prepared by the rapid relaxation of the bright state, but the "quality" of the superpositions that are formed degrades with increasing coupling strength. This degradation is already suggested by the

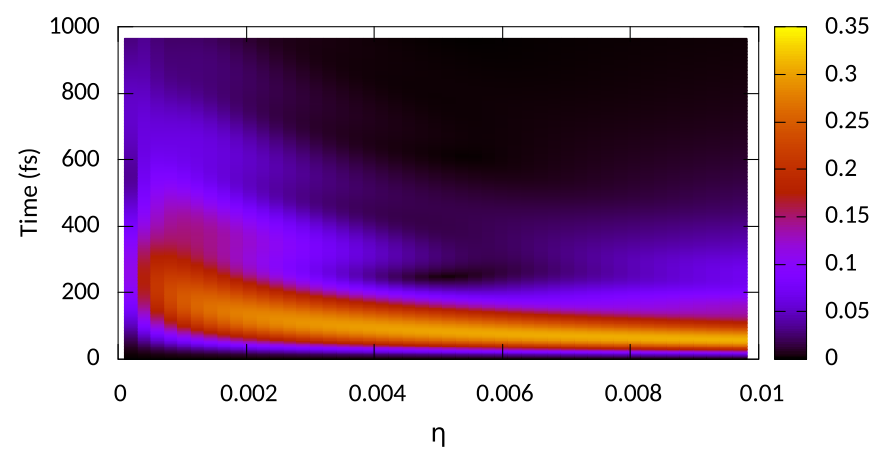

FIG. 12. Isovalue contours in the modulus of the coherence between the doublet states $\rho_{D_{+} D_{-}}(t)$ for different $\eta$ parameters [Eq. (4)] at $T=10000 \mathrm{~K}$. trends in the Redfield rates shown in Fig. 3, and confirmed by the decreasing purity of the system after relaxation in Fig. 6. This strong-coupling effect does not occur in the classical case, as the rescaling of the system-bath coupling needed to take the high-temperature limit means that the reorganization energy is always negligible compared to the system parameters.

\section{CONCLUSIONS}

In this article, we have demonstrated that the system of bright and dark excitons realized in the qubit architecture of Ref. [31] possesses near-ideal properties with respect to the nonsecular processes that allow incoherent dynamics to generate coherent wavelike motion. By using the nonperturbative HEOM technique, we have verified that under conditions of classical noise, it should be possible to experimentally resolve these coherent dynamics through the related oscillatory motion across the network in real space, although the effectively high ("infinite") temperature of the classical stochastic bath leads to strong dephasing via incoherent "uphill" population transitions from the dark manifold. Nevertheless, a previously unanticipated prediction arises from this fact and is seen in our simulations: the periodic violation of detailed balance caused by the suppression of uphill transitions as the excitation moves coherently away from the site-local source of the noise. At the same time, the existence of resolved quantum oscillations also appears to be sensitive to the correlation time of the environment, with nonperturbative theories showing stronger coherent dynamics at the same coupling strength as a simple Redfield approach. The differences persist over the correlation time of the environment, and it may be possible that this is related to the finite "switch on" time of the rapid transitions that are present from $t=0$ in the Markovian theory. Although, in general, these effects do not correlate with any obvious feature in the measure of non-Markovianity, it is clear that "memory" or temporally nonlocal effects constitute another handle by which coherent energy transfer might be manipulated. Indeed, the real-time switch from a weaker to stronger dissipative coupling might be more generally important for coherent realspace motion, as suggested for quasicoherent charge separation in organic bulk heterojunctions [1,28,80,81].

In the case of quantum noise, which might be realized by using the multilevel nature of superconducting qubits to simulate a quantum harmonic oscillator [82], the longevity of the superposition states is only limited by the form of the spectral density and the strength of interactions at the small energy gap between the dark doublet of states, or at zero frequency (pure dephasing). Spectral functions that vanish rapidly at low frequencies while having large amplitudes at the much larger bright-dark energy gap would therefore be advantageous for coherence generation. However, the coherent dynamics are suppressed at very strong coupling-regardless of the shape of the spectral density-by the growing reorganization energy of the environment, which detunes and localizes the lowlying excitations. Finally, our physical understanding of the numerical results has often relied on predictions from Redfield theory that arise from the site-local noise in our model. Given the capabilities of present simulators to apply site-specific noises, it would be very interesting in the future to consider how 
applying different spatial and spectral correlations to noises impacts coherent dynamics in quantum energy transfer networks.

\section{ACKNOWLEDGMENTS}

We are grateful to Anton Potočnik for helpful discussions and suggestions. A.C. acknowledges support from the Jean D'Alembert Chaire from IDEX Paris-Saclay, Contract No. CNRS 157819. E.M. acknowledges support from the ANRDFG COQS, under Grant No. ANR-15-CE30-0023-01. This work has been performed within the French GDR 3575 THEMS and we thank Olivier Dulieu for his support.

\section{APPENDIX A: NON-MARKOVIANITY ANALYSIS}

We briefly discuss the non-Markovianity of the dynamics for some coupling ranges. Numerous non-Markovianity witnesses have been proposed in the literature [83-85], but here we consider only the volume of accessible states in the generalized Bloch sphere [78]. Equations (14) define a time-local dynamical map $\rho_{S}(t)=\phi_{t}\left[\rho_{S}(0)\right]$ which may be expressed in an operator basis set $\left\{G_{m}\right\}$ for the Liouville space of dimension $d^{2}$ by a generalization of the Pauli matrices for $d=3$. The expansion of the matrix density in this basis leads to the Bloch representation of the system. In matrix form, the map reads $F_{m, n}(t)=\operatorname{Tr}\left(G_{m} \phi_{t}\left[G_{n}\right]\right)$ and the volume of accessible states in the Bloch sphere can be obtained from the determinant of this matrix,

$$
V(t)=\operatorname{det}(\mathbf{F})
$$

A nonmonotonous decrease of this volume is a signature on a non-Markovian backflow from the bath to the system. This is illustrated in Fig. 13 by the volume of the accessible states in the Bloch sphere for different $\eta$ parameters. For instance, bumps in the volume are obtained for the strong-coupling case $\eta=$ 0.16 (solid line). This justifies that dynamics must be treated beyond the Redfield approximation and probably beyond the second-order regime. The perturbative regime for $\eta<0.02$ leads to a smooth evolution of the population (see Fig. 4 or 10). On the contrary, oscillations are observed during the decay of the bright state for stronger coupling. This behavior may

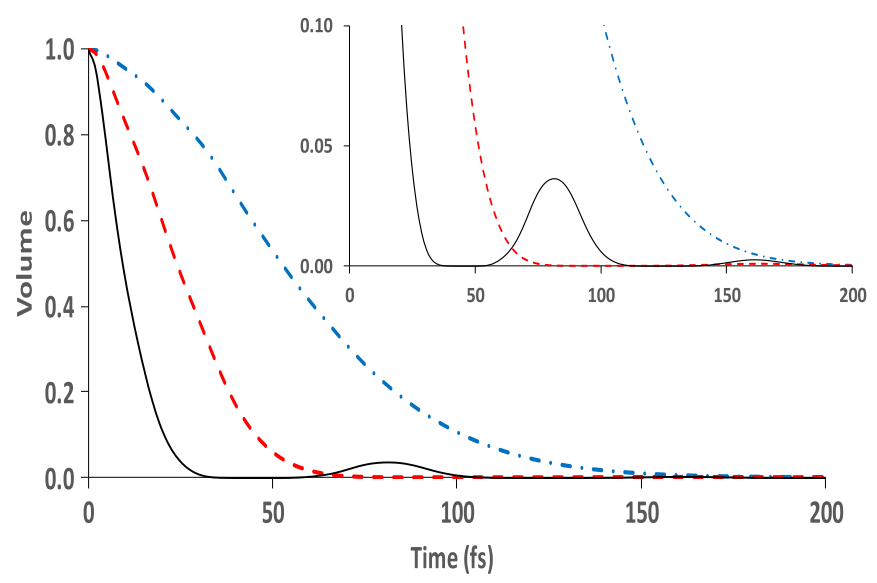

FIG. 13. Volume of the accessible state $V(t)$. Solid line: $\eta=0.16$; dashed line: $\eta=0.04$; dash-dotted line: $\eta=0.01$.

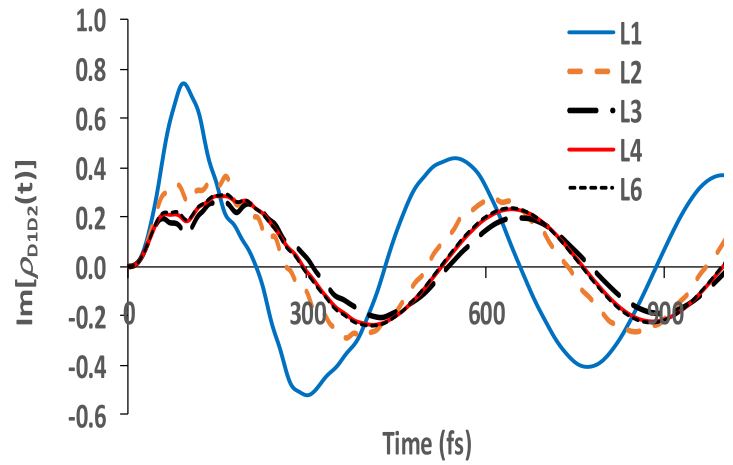

FIG. 14. Convergence of the real part of the coherence $\rho_{D_{-} D_{+}}(t)$ with respect to the level $L$ of the hierarchy corresponding to order $2 L$ in perturbation for the case $\eta=0.16$.

be related to some non-Markovian effects characterized by backflow from the environment to the system.

The level of HEOM hierarchy ensuring convergence of the simulation depends on the strength of the system-bath coupling. Level $L$ in the hierarchy corresponds to order $2 L$ in the perturbation approach. Convergence is checked in Fig. 14 by analyzing the coherence that always converges more slowly than the populations. The cases with $\eta \leqslant 0.02$ remain in the perturbative regime. On the contrary, for the case $\eta=0.16$, the regime is obviously nonperturbative and level $L=4$ is required.

We do find that although the Markovian second-order perturbative theory (Bloch-Redfield) allows an intuitive, qualitative understanding of the dynamics, it fails to describe the dynamics with quantitative accuracy (cf. HEOM results). Even at relatively weak coupling, this can lead to significant differences, as shown in Fig. 15. Given the relatively weak coupling involved, we believe that the origin of these differences is related to the Markov (time-local) approximation in Redfield theory, which does not take into account the long correlation time of the environmental spectral functions that we consider. This is supported by the results in Fig. 15, which show that the differences in dynamics become negligible for times longer than the bath correlation time $(\approx 200 \mathrm{fs})$, as shown in Fig. 2 . As the oscillatory coherent dynamics induced by the relaxation are

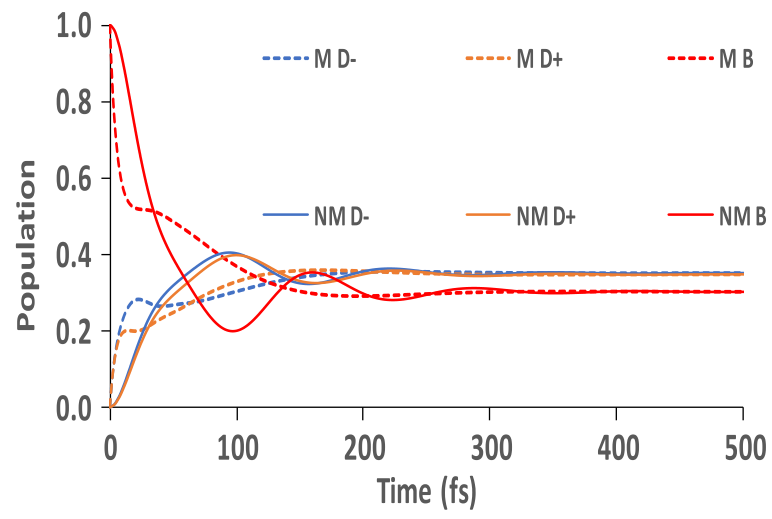

FIG. 15. Comparison of the eigenstate population dynamics computed with the Markovian (M) Redfield equations (dashed lines) and the numerically non-Markovian (NM) exact results obtained by HEOM (solid lines) at relatively weak coupling $\eta=0.01$. 
much more prominent in the HEOM results, this indicates that a proper treatment of extended bath correlation times can also be an important factor for noise-induced coherence generation in the classical case, further emphasizing the need for methods such as HEOM.

\section{APPENDIX B: PARAMETERS OF THE SPECTRAL DENSITY}

The parameters of the superohmic expression [Eq. (6)] for the thin and broad spectral density used in the HEOM
TABLE I. The parameters of the superohmic expression (6) used in the HEOM simulations.

\begin{tabular}{lccc}
\hline \hline$\Omega_{l}$ (a.u.) & $\Gamma_{1}$ (a.u.) & $\Omega_{2}$ (a.u.) & $\Gamma_{1}$ (a.u.) \\
\hline $9.562 \times 10^{-4}$ & $6.3537 \times 10^{-3}$ & $4.5639 \times 10^{-3}$ & $2.7188 \times 10^{-4}$ \\
$2.762 \times 10^{-3}$ & $1.6554 \times 10^{-3}$ & $6.4639 \times 10^{-3}$ & $2.5319 \times 10^{-3}$ \\
\hline
\end{tabular}

simulations are gathered in Table I. The $p$ parameter is taken as $1.95 \times 10^{-14} \times f$, where $f$ is adjusted to obtain the different renormalization energies.
[1] J.-L. Brédas, E. H. Sargent, and G. D. Scholes, Nat. Mater. 16, 35 (2017).

[2] G. D. Scholes, G. R. Fleming, L. X. Chen, A. Aspuru-Guzik, A. Buchleitner, D. F. Coker, G. S. Engel, R. van Grondelle, A. Ishizaki, D. M. Jonas, J. S. Lundeen, J. K. McCusker, S. Mukamel, J. P. Ogilvie, A. Olaya-Castro, M. A. Ratner, F. C. Spano, K. B. Whaley, and X. Zhu, Nature (London) 543, 647 (2017).

[3] E. J. O'Reilly and A. Olaya-Castro, Nat. Commun. 5, 3012 (2014).

[4] A. Chin, J. Prior, R. Rosenbach, F. Caycedo-Soler, S. Huelga, and M. Plenio, Nat. Phys. 9, 113 (2013).

[5] E. Collini, C. Y. Wong, K. E. Wilk, P. M. G. Curmi, P. Brumer, and G. D. Scholes, Nature (London) 463, 644 (2010).

[6] F. D. Fuller, J. Pan, A. Gelzinis, V. Butkus, S. S. Senlik, D. E. Wilcox, C. F. Yocum, L. Valkunas, D. Abramavicius, and J. P. Ogilvie, Nat. Chem. 6, 706 (2014).

[7] C. Kreisbeck and T. Kramer, J. Phys. Chem. Lett. 3, 2828 (2012).

[8] N. Lambert, Y. N. Chen, Y. C. Cheng, C. M. Li, G. Y. Chen, and F. Nori, Nat. Phys. 9, 10 (2013).

[9] H. Lee, Y.-C. Cheng, and G. R. Fleming, Science 316, 1462 (2007).

[10] G. Panitchayangkoon, D. Hayes, K. A. Fransted, J. R. Caram, E. Harel, J. Wen, R. E. Blankenship, and G. S. Engel, Proc. Natl. Acad. Sci. USA 107, 12766 (2010).

[11] E. Romero, R. Augulis, V. I. Novoderezhkin, M. Ferretti, J. Thieme, D. Zigmantas, and R. Van Grondelle, Nat. Phys. 10, 676 (2014).

[12] A. Ishizaki and G. R. Fleming, Proc. Natl. Acad. Sci. USA 106, 17255 (2009).

[13] H.-B. Chen, N. Lambert, Y.-C. Cheng, Y.-N. Chen, and F. Nori, Sci. Rep. 5, 12753 (2015).

[14] A. W. Chin, S. F. Huelga, and M. B. Plenio, Philos. Trans. R. Soc. A 370, 3638 (2012).

[15] A. G. Dijkstra and Y. Tanimura, Phys. Rev. Lett. 104, 250401 (2010).

[16] E. K. Irish, R. Gómez-Bombarelli, and B. W. Lovett, Phys. Rev. A 90, 012510 (2014).

[17] J. Iles-Smith, A. G. Dijkstra, N. Lambert, and A. Nazir, J. Chem. Phys. 144, 044110 (2016).

[18] N. Killoran, S. F. Huelga, and M. B. Plenio, J. Chem. Phys. 143, 155102 (2015).

[19] P. Malý, O. J. G. Somsen, V. I. Novoderezhkin, T. Mančal, and R. van Grondelle, ChemPhysChem 17, 1356 (2016).
[20] M. Qin, H. Z. Shen, X. L. Zhao, and X. X. Yi, Phys. Rev. A 96, 012125 (2017).

[21] D. H. Santamore, N. Lambert, and F. Nori, Phys. Rev. B 87, 075422 (2013).

[22] R. Stones and A. Olaya-Castro, Chem. 1, 822 (2016).

[23] A. A. Bakulin, S. E. Morgan, T. B. Kehoe, M. W. Wilson, A. W. Chin, D. Zigmantas, D. Egorova, and A. Rao, Nat. Chem. 8, 16 (2016).

[24] S. M. Falke, C. A. Rozzi, D. Brida, M. Maiuri, M. Amato, E. Sommer, A. De Sio, A. Rubio, G. Cerullo, E. Molinari et al., Science 344, 1001 (2014).

[25] J. Lim, D. Paleček, F. Caycedo-Soler, C. N. Lincoln, J. Prior, H. Von Berlepsch, S. F. Huelga, M. B. Plenio, D. Zigmantas, and J. Hauer, Nat. Commun. 6, 7755 (2015).

[26] F. Novelli, A. Nazir, G. H. Richards, A. Roozbeh, K. E. Wilk, P. M. Curmi, and J. A. Davis, J. Phys. Chem. Lett. 6, 4573 (2015).

[27] É. Boulais, N. P. Sawaya, R. Veneziano, A. Andreoni, J. L. Banal, T. Kondo, S. Mandal, S. Lin, G. S. Schlau-Cohen, N. W. Woodbury et al., Nat. Mater. 17, 159 (2018).

[28] S. Gélinas, A. Rao, A. Kumar, S. L. Smith, A. W. Chin, J. Clark, T. S. van der Poll, G. C. Bazan, and R. H. Friend, Science 343, 512 (2014).

[29] E. A. Hemmig, C. Creatore, B. Wu nsch, L. Hecker, P. Mair, M. A. Parker, S. Emmott, P. Tinnefeld, U. F. Keyser, and A. W. Chin, Nano Lett. 16, 2369 (2016).

[30] H.-G. Duan, V. I. Prokhorenko, R. J. Cogdell, K. Ashraf, A. L. Stevens, M. Thorwart, and R. D. Miller, Proc. Natl. Acad. Sci. USA 114, 8493 (2017).

[31] A. Potočnik, A. Bargerbos, F. A. Schröder, S. A. Khan, M. C. Collodo, S. Gasparinetti, Y. Salathé, C. Creatore, C. Eichler, H. E. Türeci et al., Nat. Commun. 9, 904 (2018).

[32] V. May et al., Charge and Energy Transfer Dynamics in Molecular Systems (Wiley, New York, 2008).

[33] T. Renger, V. May, and O. Kühn, Phys. Rep. 343, 137 (2001).

[34] G. D. Scholes, G. R. Fleming, A. Olaya-Castro, and R. Van Grondelle, Nat. Chem. 3, 763 (2011).

[35] P. Rebentrost, M. Mohseni, I. Kassal, S. Lloyd, and A. AspuruGuzik, New J. Phys. 11, 33003 (2009).

[36] F. Caruso, A. W. Chin, A. Datta, S. F. Huelga, and M. B. Plenio, J. Chem. Phys. 131, 105106 (2009).

[37] M. B. Plenio and S. F. Huelga, New J. Phys. 10, 113019 (2008).

[38] A. W. Chin, A. Datta, F. Caruso, M. B. Plenio, and S. F. Huelga, New J. Phys. 12, 065002 (2010). 
[39] H.-B. Chen, P.-Y. Chiu, and Y.-N. Chen, Phys. Rev. E 94, 052101 (2016).

[40] S. F. Huelga and M. B. Plenio, Contemp. Phys. 54, 181 (2013).

[41] J. Schulze and O. Kuhn, J. Phys. Chem. B 119, 6211 (2015).

[42] I. de Vega and D. Alonso, Rev. Mod. Phys. 89, 015001 (2017).

[43] A. Fruchtman, N. Lambert, and E. M. Gauger, Sci. Rep. 6, 28204 (2016).

[44] K. H. Hughes, C. D. Christ, and I. Burghardt, J. Chem. Phys. 131, 024109 (2009).

[45] A. W. Chin, Á. Rivas, S. F. Huelga, and M. B. Plenio, J. Math. Phys. 51, 092109 (2010).

[46] J. Prior, A. W. Chin, S. F. Huelga, and M. B. Plenio, Phys. Rev. Lett. 105, 050404 (2010).

[47] N. Makri, J. Phys. Chem. A 102, 4414 (1998).

[48] U. Manthe, J. Chem. Phys. 128, 164116 (2008).

[49] R. Martinazzo, B. Vacchini, K. H. Hughes, and I. Burghardt, J. Chem. Phys. 134, 011101 (2011).

[50] P. Nalbach, A. Ishizaki, G. R. Fleming, and M. Thorwart, New J. Phys. 13, 063040 (2011).

[51] J. Prior, I. de Vega, A. W. Chin, S. F. Huelga, and M. B. Plenio, Phys. Rev. A 87, 013428 (2013).

[52] F. A. Schröder, D. H. Turban, A. J. Musser, N. D. Hine, and A. W. Chin, arXiv:1710.01362.

[53] P. Strasberg, G. Schaller, N. Lambert, and T. Brandes, New J. Phys. 18, 073007 (2016).

[54] Y. Tanimura and R. Kubo, J. Phys. Soc. Jpn. 58, 101 (1989).

[55] M. Thoss, H. Wang, and W. H. Miller, J. Chem. Phys. 115, 2991 (2001).

[56] A. Ishizaki and Y. Tanimura, J. Phys. Soc. Jpn. 74, 3131 (2005).

[57] Y. Tanimura, J. Phys. Soc. Jpn. 75, 082001 (2006).

[58] R.-X. Xu and Y. J. Yan, Phys. Rev. E 75, 031107 (2007).

[59] J. Strümpfer and K. Schulten, J. Chem. Theory Comput. 8, 2808 (2012).

[60] A. Ishizaki and G. R. Fleming, J. Chem. Phys. 130, 234111 (2009).

[61] Q. Shi, L. Chen, G. Nan, R.-X. Xu, and Y. Yan, J. Chem. Phys. 130, 084105 (2009).

[62] A. Dodin, T. V. Tscherbul, and P. Brumer, J. Chem. Phys. 144, 244108 (2016).

[63] A. C. Han, M. Shapiro, and P. Brumer, J. Phys. Chem. A 117, 8199 (2013).

[64] D. J. Gorman, B. Hemmerling, E. Megidish, S. A. Moeller, P. Schindler, M. Sarovar, and H. Haeffner, Phys. Rev. X 8, 011038 (2018).
[65] C. Creatore, M. A. Parker, S. Emmott, and A. W. Chin, Phys. Rev. Lett. 111, 253601 (2013).

[66] K. E. Dorfman, D. V. Voronine, S. Mukamel, and M. O. Scully, Proc. Natl. Acad. Sci. USA 110, 2746 (2013).

[67] D. Gelbwaser-Klimovsky and A. Aspuru-Guzik, Chem. Sci. 8, 1008 (2017).

[68] D. Newman, F. Mintert, and A. Nazir, Phys. Rev. E 95, 032139 (2017).

[69] M. O. Scully, Phys. Rev. Lett. 104, 207701 (2010).

[70] Y. Zhang, S. Oh, F. H. Alharbi, G. S. Engel, and S. Kais, Phys. Chem. Chem. Phys. 17, 5743 (2015).

[71] F. P. Heinz-Peter Breuer, The Theory of Open Quantum Systems (Oxford University Press, Oxford, 2002).

[72] K. Blum, Density Matrix Theory and Applications (Springer Science \& Business Media, New York, 2013).

[73] J. Jeske, D. J. Ing, M. B. Plenio, S. F. Huelga, and J. H. Cole, J. Chem. Phys. 142, 064104 (2015).

[74] P. R. Eastham, P. Kirton, H. M. Cammack, B. W. Lovett, and J. Keeling, Phys. Rev. A 94, 012110 (2016).

[75] H. Liu, L. Zhu, S. Bai, and Q. Shi, J. Chem. Phys. 140, 134106 (2014).

[76] E. Mangaud, C. Meier, and M. Desouter-Lecomte, Chem. Phys. 494, 90 (2017).

[77] A. Pomyalov, C. Meier, and D. Tannor, Chem. Phys. 370, 98 (2010).

[78] S. Lorenzo, F. Plastina, and M. Paternostro, Phys. Rev. A 84, 032124 (2011).

[79] S. Oviedo-Casado, J. Prior, A. W. Chin, R. Rosenbach, S. F. Huelga, and M. B. Plenio, Phys. Rev. A 93, 020102(R) (2016).

[80] S. L. Smith and A. W. Chin, Phys. Chem. Chem. Phys. 16, 20305 (2014).

[81] S. L. Smith and A. W. Chin, Phys. Rev. B 91, 201302 (2015)

[82] S. Mostame, P. Rebentrost, A. Eisfeld, A. J. Kerman, D. I. Tsomokos, and A. Aspuru-Guzik, New J. Phys. 14, 105013 (2012).

[83] H.-P. Breuer, E.-M. Laine, and J. Piilo, Phys. Rev. Lett. 103, 210401 (2009).

[84] E.-M. Laine, J. Piilo, and H.-P. Breuer, Phys. Rev. A 81, 062115 (2010).

[85] M. J. W. Hall, J. D. Cresser, L. Li, and E. Andersson, Phys. Rev. A 89, 042120 (2014). 